\title{
Influência do posicionamento radiográfico no cálculo interobservador do ângulo do platô tibial em cães
}

[Influence of radiographic positioning in the interobserver measuring of the tibial plateau angle in dogs]

\author{
S.M. Capelasso ${ }^{1}$, R. Zanatta ${ }^{1}$, R.S. Lemos $^{1}$, M.D. Santos ${ }^{1}$, F.M. Silva ${ }^{1}$, S.H. Freitas ${ }^{1}$, \\ A.M. Amude ${ }^{1}$, C.R.A. Ferrigno ${ }^{2}$, N.M.B. Dower ${ }^{3}$, K.C.I. Yamauchi ${ }^{1}{ }^{*}$ \\ 1Universidade de Cuiabá - UNIC - Cuiabá, MT \\ ${ }^{2}$ Universidade de São Paulo - USP- São Paulo, SP \\ ${ }^{3}$ Universidade do Mato Grosso - UFMT - Cuiabá, MT
}

\begin{abstract}
RESUMO
O objetivo deste trabalho foi demonstrar que o posicionamento radiográfico inadequado do membro pélvico de cães (imagens obliquadas) afeta a mensuração do ângulo do platô tibial (APT), levando à maior variação entre observadores. Foram realizadas duas projeções radiográficas nos membros pélvicos direito e esquerdo, uma em perfil verdadeiro e a outra com o membro obliquado em 21 cães provenientes do atendimento clínico cirúrgico do Hospital Veterinário da Universidade de Cuiabá, em Cuiabá-MT. Os critérios de inclusão foram cães livres de histórico ou afecção ortopédica nos membros pélvicos, pesando entre 15 e 40 quilos, sem predileção por raça ou sexo, entre dois e sete anos de idade. Foram obtidas 42 imagens radiográficas em perfil verdadeiro e 42 imagens radiográficas obliquadas. Três observadores com experiência em cálculo do APT fizeram as mensurações. Nos cálculos das imagens em perfil verdadeiro, o observador 1 obteve uma média de $23,35^{\circ} \pm 2,82$; o observador 2 , média de $23,88^{\circ} \pm 4,83$; e o observador 3 , média de $24,54^{\circ} \pm 4,01$, não sendo encontrada diferença significativa entre o cálculo dos três avaliadores, $\mathrm{P}=0,944(\mathrm{P}>0,05)$, nas imagens em perfil verdadeiro. Nos cálculos das imagens obliquadas, o observador 1 obteve uma média de $21,69^{\circ} \pm 4,22$; o observador 2 , média de $19,42^{\circ} \pm 5,92$; e o observador 3, média de $22,64^{\circ} \pm 5,23$, e foi encontrada diferença significativa entre o cálculo dos três observadores, $\mathrm{P}=0,016(\mathrm{P}<0,05)$. Com esses dados, pôde-se demonstrar que a imagem radiográfica obliquada compromete o valor final do cálculo do ângulo do platô tibial em cães, levando à variação numérica estatisticamente significativa entre observadores. Conclui-se, assim, que um posicionamento correto do membro pélvico, em perfil verdadeiro, deve ser sempre utilizado para a obtenção de imagens, com o objetivo de mensurar o valor do APT.
\end{abstract}

Palavras-chave: membro pélvico, ligamento cruzado cranial, osteotomias, posicionamento, ruptura

\begin{abstract}
This work aimed at demonstrating that an inadequate radiographic position of pelvic limbs of dogs, from a strict lateral radiographic view, resulted in oblique images, affecting the measurement of the tibial plateau angle (TPA). There were two radiographic projections of the right and the left pelvic limb, one in strict lateral radiographic view and another with inclined hind limb in 21 dogs from the clinical surgical assistance at Hospital Veterinário of Universidade de Cuiabá in Cuiabá-MT. Inclusion criteria were dogs free from pelvic hind limbs orthopedic affection records, weighing between 15 and 40 kilos, without a preference for breed or gender and ageing between two and seven years old. A total of 42 radiographic images in strict lateral radiographic view and 42 inclined limb radiographic views were obtained. Three observers with TPA calculation experience made measurements. In the calculus for the strict lateral radiographic view the first observer has reached an average of $23,35^{\circ} \pm 2,82$, the second observer $23,88^{\circ}$
\end{abstract}

Recebido em 14 de julho de 2016

Aceito em 3 de novembro de 2016

*Autor para correspondência (corresponding author)

E-mail: itokelly@yahoo.com.br 
$\pm 4,83$ and the third observer $24,54^{\circ} \pm 4,01$. No significant statistic differences among the calculus of the three evaluators $p=0,944(P>0,05)$ was found. In the calculus of the inclined limbs radiographic views the first observer reached an average of $21,69^{\circ} \pm 4,22$, the second observer $19,42^{\circ} \pm 5,92$ and the third observer $22,64^{\circ} \pm 5,23$. Significant statistic difference in the calculus of the three observers, $p=0,016$, that is to say, $P<0,05$ was found. The data indicated that the inclined limb radiographic image compromises the final value of the calculation of tibial plateau angle in dogs. Thus, correct positioning of the pelvic limb, in a strict lateral radiographic view, must be always used for obtaining images aiming at measuring the value of TPA.

Keywords: pelvic limbs, cruciate ligaments, osteotomy, positioning, rupture

\section{INTRODUÇÃO}

A ruptura do ligamento cruzado cranial (RLCCr) resulta em instabilidade rotacional e translacional da articulação do joelho canino, que leva ao desenvolvimento de osteoartrose (Vasseur e Berry, 1992). Várias são as etiologias da RLCCr, e a sua patogênese exata ainda não foi determinada, podendo ser causada por traumas, processos degenerativos (Vasseur et al., 1985), doenças autoimunes (Arnoczky, 1996) e uma inclinação excessiva do ângulo do platô tibial (Reif e Probst, 2003; Zeltzman et al., 2005; Osmond et al., 2006).

As osteotomias corretivas se enquadram nas técnicas mais atuais para tratamento da RLCCr em cães. Elas promovem estabilidade funcional do joelho durante a fase do passo, reduzem o deslocamento de translação cranial da tíbia, conforme novo conceito de tratamento, que preconiza estabilidade dinâmica, alterando a geometria óssea da articulação (Kim et al., 2008). Para a escolha do melhor tratamento da RLCCr, em cães, dentro das técnicas de osteotomias corretivas, a mensuração do ângulo do platô tibial (APT) é um importante componente a ser determinado (Reif et al., 2004).

A técnica de mensuração do APT foi descrita por Slocum e Devine (1983) e é realizada por meio de imagens radiográficas da tíbia, em incidência mediolateral em perfil verdadeiro, e consiste em traçar três linhas: a primeira linha no eixo mecânico da tíbia, que vai do centro do tarso até a eminência intercondilar; a segunda linha é traçada unindo o ponto mais cranial ao ponto mais caudal da superfície do platô tibial e, por último, uma terceira linha é traçada perpendicular ao eixo longo da tíbia, no ponto de intersecção das duas primeiras linhas. O APT é formado pela junção da segunda e terceira linhas.
O posicionamento da tíbia durante o exame radiográfico pode influenciar na aparência dos pontos de referência para determinação do APT e induzir a variação do valor do APT exato, comprometendo a escolha da melhor técnica cirúrgica ou o sucesso cirúrgico dela (Reif et al., 2004). Diante da possibilidade de variação do valor exato do APT em caso de posicionamento radiográfico inadequado, comprometendo $\mathrm{o}$ resultado das osteotomias corretivas, este trabalho propõe avaliar a influência do posicionamento radiográfico obliquado no cálculo do APT em cães, uma vez que o ângulo calculado errado pode levar a mau planejamento e a não resolução dos sinais clínicos manifestados na ruptura de ligamento cruzado cranial em cães.

\section{MATERIAL E MÉTODOS}

Foi realizado estudo prospectivo, em cães provenientes do atendimento clínico cirúrgico do Hospital Veterinário da Universidade de Cuiabá - Hovet. O trabalho foi submetido à Comissão de Ética de Uso de Animais da Universidade de Cuiabá e aprovado, protocolado sob o número 012/2014. As imagens radiográficas foram realizadas e avaliadas no Setor de Radiologia do Hospital Veterinário da Universidade de Cuiabá (Hovet-Unic).

Foram radiografados 21 animais, sem predileção por raça ou sexo, livres de histórico ou afecção ortopédica em membros pélvicos que comprometeriam o cálculo do ângulo do platô tibial (fraturas de côndilos femorais ou tibiais, trocleoplastia, deformidade tibial secundária a má união), pesando entre 15 e 40 quilos, acima de dois anos de idade. Todos os animais foram sedados/anestesiados para o procedimento radiográfico adequado. $\mathrm{O}$ protocolo utilizado para sedação foi o cloridrato de petidina, na dose 
de $4 \mathrm{mg} / \mathrm{kg}$, associado à acepromazina, na dose de $0,5 \mathrm{mg} / \mathrm{kg}$, por via intramuscular. Após 20 minutos, aqueles animais que não permitiram o posicionamento adequado com esse protocolo de sedação foram submetidos à anestesia geral com propofol, na dose de $5 \mathrm{mg} / \mathrm{kg}$, por via intravenosa. $\mathrm{O}$ exame radiográfico foi realizado por meio do aparelho de raios $\mathrm{X}$, marca SAWAE, modelo Altus ST503-HF. Foram obtidas radiografias dos membros pélvicos direito e esquerdo de cada animal, em projeção mediolateral, conforme metodologia estabelecida por Slocum e Devine (1983), em duas projeções, perfil lateral verdadeiro, caracterizada pela sobreposição dos côndilos femorais e tibiais, e perfil lateral obliquado, caracterizada pela não sobreposição dos côndilos femorais e tibiais. Nas radiografias, foram incluídas as articulações do joelho e do tarso. Obtidas as radiografias, três avaliadores com experiência em radiologia realizaram manualmente o cálculo do APT, o qual foi mensurado com base na imagem radiográfica, utilizando-se como pontos de referência o centro do tarso, as eminências intercondíleas da tíbia e as extremidades cranial e caudal da superfície do platô tibial, de acordo com técnica descrita por Slocum e Devine (1983) (Fig. 1).

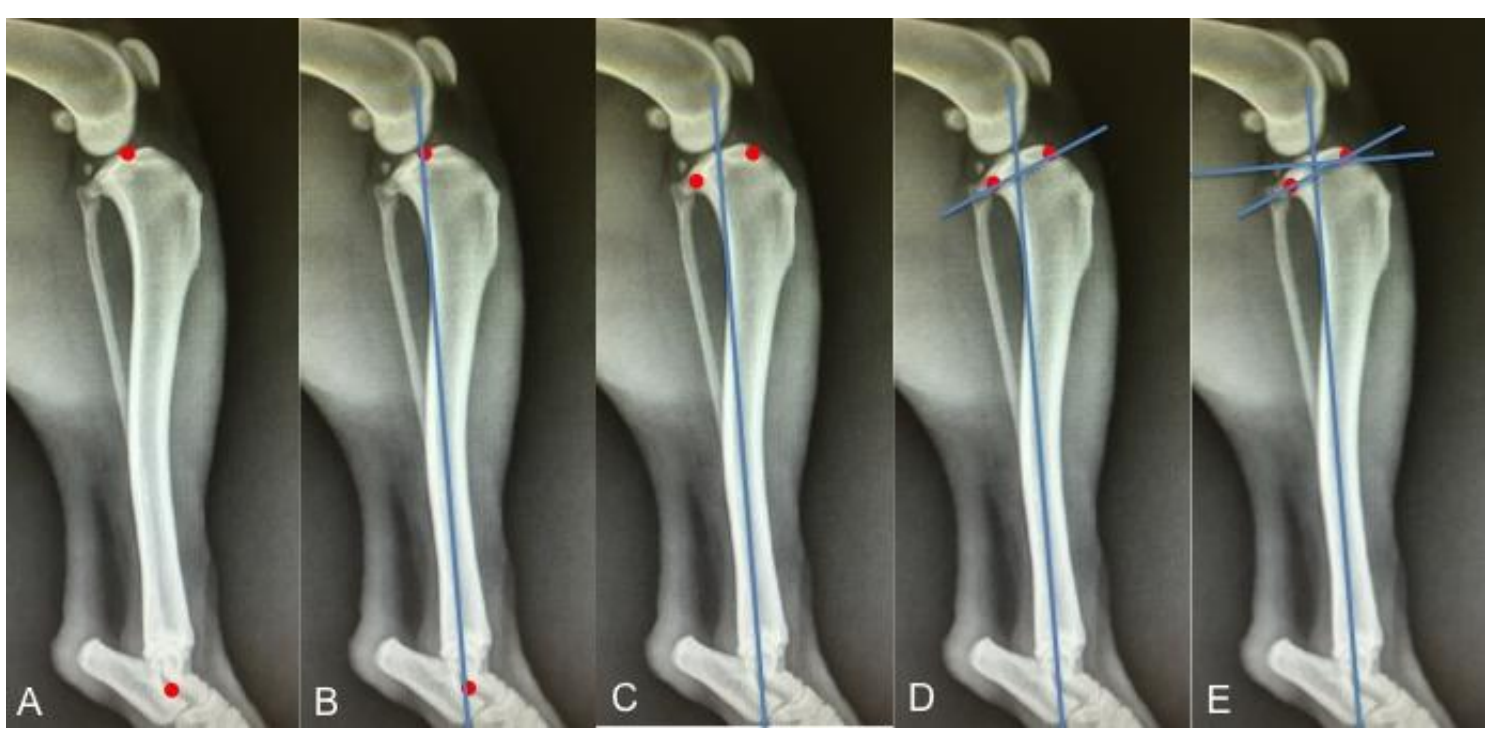

Figura 1. Imagem radiográfica de tíbia de cão em perfil verdadeiro e sequência da localização dos pontos de referência e traçado de linhas para cálculo do ângulo do platô tibial. (A) Localização das eminências intercondíleas da tíbia no platô tibial e do centro do tarso. (B) Linha 1 unindo eminências intercondíleas e centro do tarso (eixo mecânico da tíbia). (C) Localização das extremidades cranial e caudal da superfície do platô tibial. (D) Linha 2 unindo pontos do platô tibial. (E) Confecção da linha 3, perpendicular ao eixo mecânico da tíbia na intersecção das linhas 1 e 2. O ângulo do platô tibial é obtido entre as linhas 2 e 3.

Cada observador analisou dois grupos. O grupo um foi constituído por 42 valores do APT das imagens radiográficas em perfil lateral verdadeiro, sem distinção de membro direito ou esquerdo; o grupo dois foi constituído por 42 valores do APT das imagens radiográficas obliquadas, sem distinção de membro direito ou esquerdo.

Os resultados foram analisados por meio do programa computacional SAEG (Sistema..., 2007), por análise de variância, e as médias comparadas pelo teste de Duncan a $5 \%$ de probabilidade.
Foram comparadas as médias das avaliações do APT, em perfil e oblíqua, dos três observadores, utilizando-se nível de significância de 5\% para todos os testes realizados.

\section{RESULTADOS}

Nos cálculos das imagens em perfil verdadeiro, os observadores obtiveram as respectivas médias: observador $1: 23,35^{\circ} \pm 2,82$, observador 2: $23,88^{\circ} \pm 4,83$ e observador $3: 24,54^{\circ} \pm 4,01$, não sendo encontrado diferença estatisticamente significativa entre o cálculo dos três avaliadores, 
$\mathrm{P}=0,944$. Nos cálculos das imagens oblíquas, os observadores obtiveram as respectivas médias: observador 1: $21,69^{\circ} \pm 4,22$, observador 2: $19,42^{\circ} \pm 5,92$ e observador $3: 22,64^{\circ} \pm 5,23$, sendo encontrada diferença estatisticamente significativa entre o cálculo dos três avaliadores, $\mathrm{P}=0,016$.

\section{DISCUSSÃO}

A escolha do tema proposto por este trabalho deu-se pelo fato de a RLCCr ser uma das afecções mais comuns em cães (Johnson e Johnson, 1993; Baraúna Júnior e Tudury, 2007; Matera et al., 2007), sendo o tratamento cirúrgico o mais recomendado para a maioria dos casos (Vasseur et al., 1985). As osteotomias corretivas são as técnicas mais atuais e, para o planejamento adequado dessas técnicas, a mensuração correta do APT é um fator que determina o sucesso desse tratamento cirúrgico (Slocum e Slocum, 1993).

O critério de inclusão de cães pesando entre 15 e $40 \mathrm{~kg}$ foi determinado com base em estudos que comprovam que essa afecção, apesar de acometer cães de pequeno porte, é mais frequente em cães de grande porte e obesos (Vasseur et al., 1985; Duval et al., 1999; Matera et al., 2007).

A seleção de animais acima de dois anos e livre de doenças degenerativas visou evitar alterações das referências anatômicas utilizadas no cálculo, e a escolha de todos os observadores com experiência em cálculo do APT objetivou homogeneizar os resultados, tendo em vista que os observadores sem experiência podem mensurar o APT erroneamente, corroborando Grierson et al. (2011) e Tartarunas et al. (2008).

As imagens foram obtidas em animais vivos, sedados ou anestesiados. A sedação foi necessária, pois facilita a manipulação do animal e sua total imobilização, visando obter imagens de qualidade em perfil verdadeiro sem a necessidade de repetições desnecessárias, o que evita a exposição do animal a maiores doses de radiação, conforme preconiza Ginja e Ferreira (2002). Ainda assim, por serem animais vivos, não foi possível definir o grau de obliquidade de cada imagem.
Não houve diferença significativa na comparação da média do APT, entre os observadores, das imagens em perfil verdadeiro, pois a experiência deles no cálculo do APT, a facilidade da visualização, dos pontos de referência para os cálculos e a padronização da técnica do cálculo preconizada por Slocum e Devine (1983) facilitaram o cálculo e possivelmente fizeram com que os observadores não fossem induzidos ao erro. Na comparação da média do APT, das imagens obliquas, houve diferença significativa entre os observadores, confirmando a hipótese deste trabalho. A imagem oblíqua altera os pontos de referência, dificultando sua localização e, mesmo com a experiência dos observadores e a facilidade da técnica de cálculo, não foi possível haver homogeneidade na média das mensurações.

\section{CONCLUSÃO}

Concluiu-se, com base nos resultados obtidos neste trabalho, que um posicionamento oblíquo da tíbia leva a uma variação significativa da mensuração do ângulo do platô tibial em cães entre observadores e que um posicionamento correto do membro pélvico, em perfil verdadeiro, deve ser sempre utilizado para a obtenção de imagens para planejamento correto das osteotomias corretivas, pois o mau planejamento pode levar a resultados cirúrgicos e clínicos insatisfatórios na correção da ruptura de ligamento cruzado cranial em cães.

\section{REFERÊNCIAS}

ARNOCZKY, S.P. Patomecânica das lesões do ligamento cruzado e meniscos. In: BOJARB, M.J. Mecanismos da moléstia na cirurgia dos pequenos animais. 3.ed. São Paulo: Manole, 1996. cap.110. p.889-902.

BARAUNA Jr., D.; TUDURY, E.A. Uso do teste de compressão tibial e do deslocamento do sesamóide poplíteo no diagnóstico radiográfico da ruptura do ligamento cruzado cranial em cães. Rev. Port. Ciênc. Vet., v.102, p.71-74, 2007.

DUVAL, J.M.; BUDSBERG, J.C.; FLO, G.L.; SAMMARCO, J.L. Breed, sex, and body weight as risk factors for rupture of the cranial cruciate ligament in young dogs. J. Am. Vet. Med. Assoc., v.215, p.811-814, 1999. 
GINJA, M.M.D.; FERREIRA, A.J.A. Efeitos biológicos da radiação $X$ e radioproteção em medicina veterinária. Rev. Port. Ciênc. Vet., v.97, p.101-109, 2002.

GRIERSON, J.; ASHER, L.; GRAINGER, K. An investigation into risk factors for bilateral canine cruciate ligament rupture. Vet. Comp. Orthop. Traumatol., v.24, p.192-196, 2011.

JOHNSON, J.M.; JOHNSON, A.L. Cranial cruciate ligament rupture: pathogenesis, diagnosis, and postoperative rehabilitation. Vet. Clin. N. Am. Small Anim. Pract., v.23, p.717733, 1993.

KIM, S.E.; POZZI, A.; KOWALESKI, M.P.; LEWIS, D.D. Tibial osteotomies for cranial cruciate ligament insufficiency in dogs. Vet. Surg., v.37, p.111-125, 2008.

MATERA, J.M.; TARTARUNAS, A.C.; OLIVEIRA. et al. Estudo epidemiológico retrospectivo de cães portadores de ruptura do ligamento cruzado cranial: 323 casos (1999 a 2005). Braz. J. Vet. Res. Anim. Sci., v.44, Supl. p.88-95, 2007.

OSMOND, C.S.; MARCELLIN-LITTLE, D.J.; HARRYSSON, O.L.; KIDD, L.B.

Morphometric assessment of the proximal portion of the tibia in dogs with and without cranial cruciate ligament rupture. Vet. Radiol. Ultrasound, v.47, p.136-141, 2006.

REIF, U.; DEJARDIN, L.M.; PROBST,C.W. et al. Influence of limb positioning and measurement method on the magnitude of the tibial plateau angle. Vet. Surg.,v.33, p.368-375, 2004.
REIF, U.; PROBST, C.W. Comparison of tibial plateau angles in normal and cranial cruciate deficient stifles of Labrador retrievers. Vet. Surg., v.32, p.385-389, 2003. SISTEMA para análises estatísticas-SAEG. Versão 9.1. Viçosa: UFV / Fundação Arthur Bernardes, 2007. 142p.

SLOCUM, B.; DEVINE, T. Cranial tibial thrust: a primary force in the canine stifle. J. Am. Vet. Med. Assoc., v.183, p.456-459, 1983.

SLOCUM, B.; SLOCUM, T.D. Tibial plateau leveling osteotomy for repair of cranial cruciate ligament rupture in the canine. Vet. Clin. N. Am. Small Anim. Pract., v.23,p.777-795, 1993.

TATARUNAS, A.C.; MARTINEZ A.; MATERA, J.M. Osteotomia de nivelamento do platô da tíbia. Semin. Ciênc. Agrár., v.29, p.685692, 2008.

VASSEUR, P.B.; BERRY, C.R. Progression of stifle osteoarthrosis following reconstruction of the cranial cruciate ligament in 21 dogs. J. Am. Hosp. Assoc., v.28, p.129-136, 1992.

VASSEUR, P.B.; POLL, R.R.; ARNOCZKY, S.P.; LAUER, E. Correlative biomechanical and histologic study of the cranial cruciate ligament in dogs. Am. J. Vet. Res., v.46, p.1842-1854, 1985.

ZELTZMAN, P.A.; PARÉ, B.; JOHNSON, G.M.; ZELTZMAN, V. et al. Relationship between age and tibial plateau angle in dogs with cranial cruciate rupture. J. Am. Anim. Hosp. Assoc., v.41, p.117-120, 2005. 